\title{
Are we ready to embrace novel therapeutic targets for women with AR-positive or AR-negative metastatic Triple-Negative Breast Cancer?
}

\author{
Katarzyna (Kate) Rygie ${ }^{1}$
}

Cite this article: Rygiel K: Are we ready to embrace novel therapeutic targets for women with AR-positive or ARnegative metastatic Triple-Negative Breast Cancer? Asia-Pac J Oncol 2021; 2: 17-26. https://doi.org/10.32948/ ajo.2021.07.28

\begin{abstract}
Triple-negative breast cancer (TNBC) is characterized by the absence of the estrogen receptor $(E R)$, progesterone receptor (PR), and human epidermal growth factor receptor 2 (HER2) expression. Depending on the androgen receptor (AR) expression, TNBC can be further divided into AR-positive TNBC (a "classical TNBC subtype) and AR-negative TNBC that is synonymous with a quadruple-negative breast cancer (QNBC). Since QNBC can be viewed as a distinct $B C$ subtype, it should be more precisely investigated from the diagnostic and therapeutic point of view.

Unfortunately, the scarcity of biomarkers and treatment targets makes QNBC extremely difficult to manage. Furthermore, in the absence of AR expression, many BCs often display particularly aggressive behavior, leading to adverse outcomes in the afflicted patients. Recently, some novel therapeutic targets have emerged. It is expected that targeted strategies could improve the survival of women suffering from this kind of malignancy.

This mini-review briefly outlines the main TNBC and QNBC subtypes and describes the current and future research directions in this area. It focuses on the AR expression (its presence vs. absence), and potential treatment approaches. This article also overviews certain molecular characteristics of TNBC and presents recently approved targeted therapies.
\end{abstract}

Key words triple-negative breast cancer, quadruple-negative breast cancer, androgen receptor, AR antagonists, immune checkpoint inhibitors, poly ADP-ribose polymerase inhibitors, antibody-drug conjugate

1. Department of Family Practice, Medical University of Silesia (SUM), Zabrze, Poland.

Correspondence: Katarzyna (Kate) Rygiel (Department of Family Practice, Medical University of Silesia (SUM), 41-800 Zabrze, Poland; E-mail: kasiaalpha@yahoo.co.uk). 


\section{Introduction}

Triple-negative breast cancer (TNBC), defined as the negative estrogen receptor (ER), progesterone receptor (PR), and human epidermal growth factor receptor 2 (HER2) expression, demonstrates the higher rates of relapse, greater metastatic potential, and shorter overall survival (OS), compared with other breast cancer (BC) subtypes [1]. It has been suggested that TNBC could be sub-divided into a more prevalent, androgen receptor (AR)-negative, also known as a quadruple-negative breast cancer (QNBC) subtype, and a "classical", AR-positive TNBC subtype (Figure 1) [1]. It appears that multiple, interrelated genetic and environmental factors can influence the incidence, course, and prognosis of this devastating malignancy, across different ethnic and socioeconomic populations of women [2]. In comparison to other BC subtypes, TNBC and QNBC are characterized by a more invasive tumor behavior (e.g., frequent local recurrences and distant metastases) and resistance to treatment, leading to adverse outcomes [3]. In addition, these aggressive BC subtypes have been more prevalent among women of African origin, and in premenopausal females (below 50 years of age) [2].

Moreover, some key factors related to augmented risk of TNBC and QNBC involve metabolic disorders (e.g., obesity, metabolic syndrome (MS), pre-diabetes, and diabetes mellitus type 2 (DMt2), reproductive factors (e.g., short breastfeeding period, high parity, oral contraceptive usage for over one year, and gestational diabetes), inappropriate nutrition (e.g., predominance of highly caloric and processed food, rich in saturated fats, trans-fats, and refined carbohydrates), physical inactivity, and low socioeconomic status or educational level. In particular, obesity, accompanied by metabolic derangements and pro-inflammatory status, sedentary lifestyle, and short lactation time have been associated with abnormal secretion of androgens [4]. The heterogeneous nature of TNBC and QNBC, as well as the diversity of the afflicted patient populations require comprehensive management strategies. Recently, some innovative therapeutic targets have emerged, which could possibly improve survival of many women suffering from this malignancy [5].

This mini-review briefly outlines the main TNBC and QNBC subtypes and describes the current and future research directions in this area. It focuses on the AR expression (its presence vs. absence), and potential treatment approaches. This article overviews certain molecular characteristics of TNBC and presents recently approved novel targeted therapies.

\section{Molecular subtypes of TNBC}

TNBC has been divided into the following molecular subtypes: basal-like subtypes (BL1 and BL2), mesenchymal (M), mesenchymal stem-like (MSL), immunomodulatory (IM), and luminal androgen receptor (LAR) (Figure 2) $[3,5,6]$. According to a similar categorization, the BL1 and BL2 can also be presented as basal-like immunosuppressed (BLIS), and basal-like immune activated (BLIA) subtypes [7]. It has been reported that the different TNBC subtypes revealed some important variability in response to neoadjuvant or adjuvant chemotherapy (CHT). It should be underscored that the BL1 has shown a more beneficial response to CHT than the LAR subtype [8]. On the one hand, TNBC presents a greater sensitivity to CHT than the non-TNBC subtypes. On the other hand, however, several women with advanced TNBC have been resistant to standard CHT regimen (e.g., anthracycline and taxanes) $[3,4]$. This unmet need is one of the driving forces behind searching for innovative treatment targets and compatible diagnostic tests or biomarkers $[3,9]$.

The impact of AR expression on the potential therapeutic

\section{options in TNBC}

AR can play a role of prognostic biomarker in BC [10]. According to a recent multi-institutional study, it has been revealed that ARpositive status was consistent with a more favorable prognosis among groups of women from US and Nigeria [10]. In contrast, a worse prognosis was reported among their counterparts from Ireland, Norway, and India [10]. Moreover, no prognostic value was noted in the group of women from UK [10]. Interestingly, it has been reported that the ER status affects the prognostic value of AR. For instance, the AR expression suggests a good prognosis in the ER-positive $\mathrm{BC}$, but the significance of AR expression in the ER-negative BC is still unclear [11]. Nevertheless, due to several methodological differences, and small samples examined in different studies on this subject, the prognostic value of AR in TNBC still remains undetermined, and thus, further research in this area is certainly needed, before drawing any definite conclusions.

AR is a transcription factor (TF), participating in the natural development of breast gland (via signaling at the level of steroid hormone receptor, in the cell nucleus), and also, it can contribute to $\mathrm{BC}$ initiation and progression [12]. However, the exact influence of the AR signaling on TNBC development is still not completely known. AR is expressed in about one third of TNBCs [13], and it has been noted that AR antagonists (ARA) (e.g., bicalutamide and enzalutamide), have shown some beneficial effects among patients with AR-positive TNBC $[14,15]$. In reality, a positive AR expression in the LAR TNBC subtype has been related to a greater sensitivity to ARA, while the BL1 subtype achieved the highest pathological complete response (pCR) rate, after an application of neoadjuvant chemotherapy (NAC) [6]. In contrast, the BL2 and LAR revealed the lowest $\mathrm{pCR}$ rates, after using NAC $[6,16]$. In addition, recent clinical trials that examined the ARA therapies (e.g., bicalutamide, enzalutamide, and abiraterone acetate plus prednisone) with $\mathrm{CHT}$, among women with $\mathrm{TNBC}$, have shown some beneficial effects of such treatment combinations [14, 15, 17]. Also, a neoadjuvant MDACC ARTEMIS trial (in process) has been addressing a population of patients with TNBC (in stages IIII), in whom the treatment has been adjusted, according to BC molecular profiling results [18]. For instance, in the case of positive AR expression, the participants have been receiving enzalutamide and paclitaxel (a component of the standard NAC) [18].

Notably, various AR splice variants (AR-Vs) are products of rearranging or alternative splicing of the AR transcript [19]. Importantly, the AR-Vs are characterized by an absence of the ligand-binding domain (LBD), which serves as a target for enzalutamide, and can activate target genes $[19,20]$. In fact, the $\mathrm{AR}$ and AR-V7 can differentially regulate target gene expression, depending on the recruitment of AR or its splice variant. Since it has been noted that the AR-V7 expression is related to adverse prognosis in many patients with $\mathrm{BC}$, there is a growing interest in exploration of the AR-V7, as a potential novel therapeutic target in patients with TNBC $[13,21]$.

Importantly, the AR can also govern tumor growth in some TNBC subtypes, which express the low levels of AR. For instance, the AR-positive tumor cell subpopulation may stimulate the growth of a cancer stem cell (CSC)-like cells, promoting resistance to CHT and recurrence of malignancy [22]. Therefore, the ARtargeting agents can be beneficial for patients with TNBC, even if their AR expression levels remain low (e.g., below 1\%) [22]. In fact, the use of combination of enzalutamide (ARA) and paclitaxel (CHT) can be more effective in preventing cancer recurrence (e.g., via targeting the CSC-like cells) than the application of paclitaxel alone [22].

Furthermore, low AR levels have been related to higher pCR rates in a clinical study assessing neoadjuvant cisplatin plus paclitaxel 


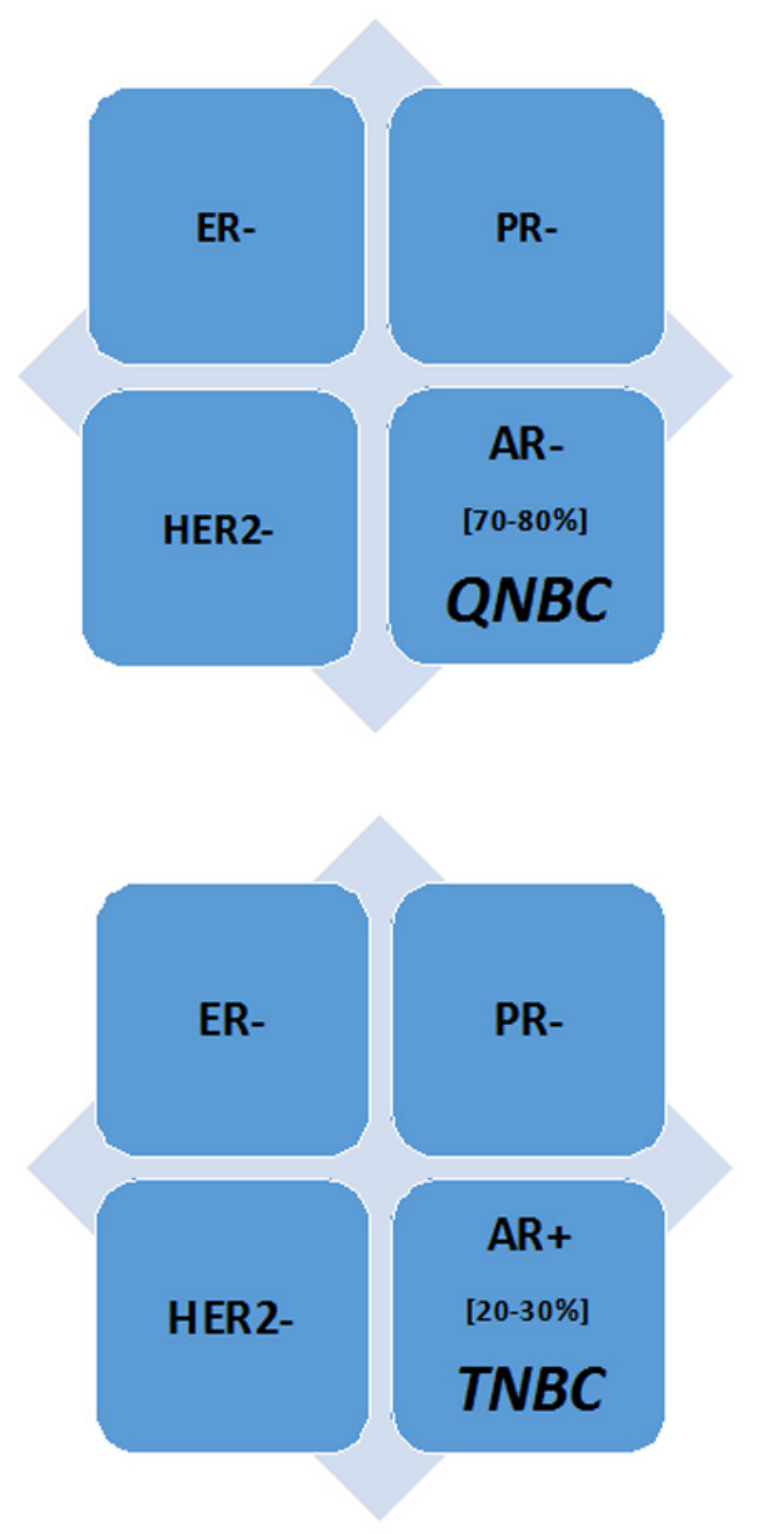

Figure 1. A receptor profile of the Triple-negative breast cancer (TNBCs) and Quadruple-negative breast cancer (QNBC) [1, 5]. Abbreviations: AR, Androgen receptor; ER, Estrogen receptor; HER2, Human epidermal growth factor receptor 2; PR; Progesterone receptor.

with or without everolimus in patients with TNBC [23]. Another study has shown that the AR expression can predict effects of therapy with tamoxifen in patients with TNBC [24]. For instance, in women with TNBC, the AR-positive status corresponded with a favorable response to tamoxifen, while the AR-negative status was consistent with a failure of the tamoxifen therapy [24].

In contrast to the ER, PR, and HER2 testing, which represent standard clinical practice, the AR testing has not been standardized due to the lack of consensus regarding its prognostic value. Since women with AR-negative TNBC (QNBC) usually suffer from a more aggressive malignancy course and worse prognosis, compared to those with AR-positive TNBC, a standardization of AR testing merits a formal resolution. The QNBC has its own specific molecular make-up, and thus, it would be beneficial to "officially" recognize it as a clinically relevant BC subtype, which is different from a "classical" TNBC [25]. At this point, immunohistochemistry (IHC) analysis of AR seems to be a feasible test to be applied for this purpose [25]. In order to develop innovative, targeted treatments for patients with ARnegative TNBC (QNBC) and AR-positive TNBC, the specific differences between these $\mathrm{BC}$ subtypes need to be explored, and then translated into targeted therapies. However, further research is still necessary in this area.

Targeted approaches to TNBC resistant to therapy - Focusing on "vulnerable spots" of aggressive malignancies

Traditionally, the standard CHT used to treat patients with highrisk and locally advanced or metastatic TNBC has been composed of anthracyclines, alkylating agents, and taxanes [9]. However, recently, some other therapeutic classes, such as the immune checkpoint inhibitors (ICI), poly ADP-ribose polymerase (PARP) inhibitors, and antibody drug conjugate (ADC), have been explored in phase III randomised controlled trials (RCTs), and 


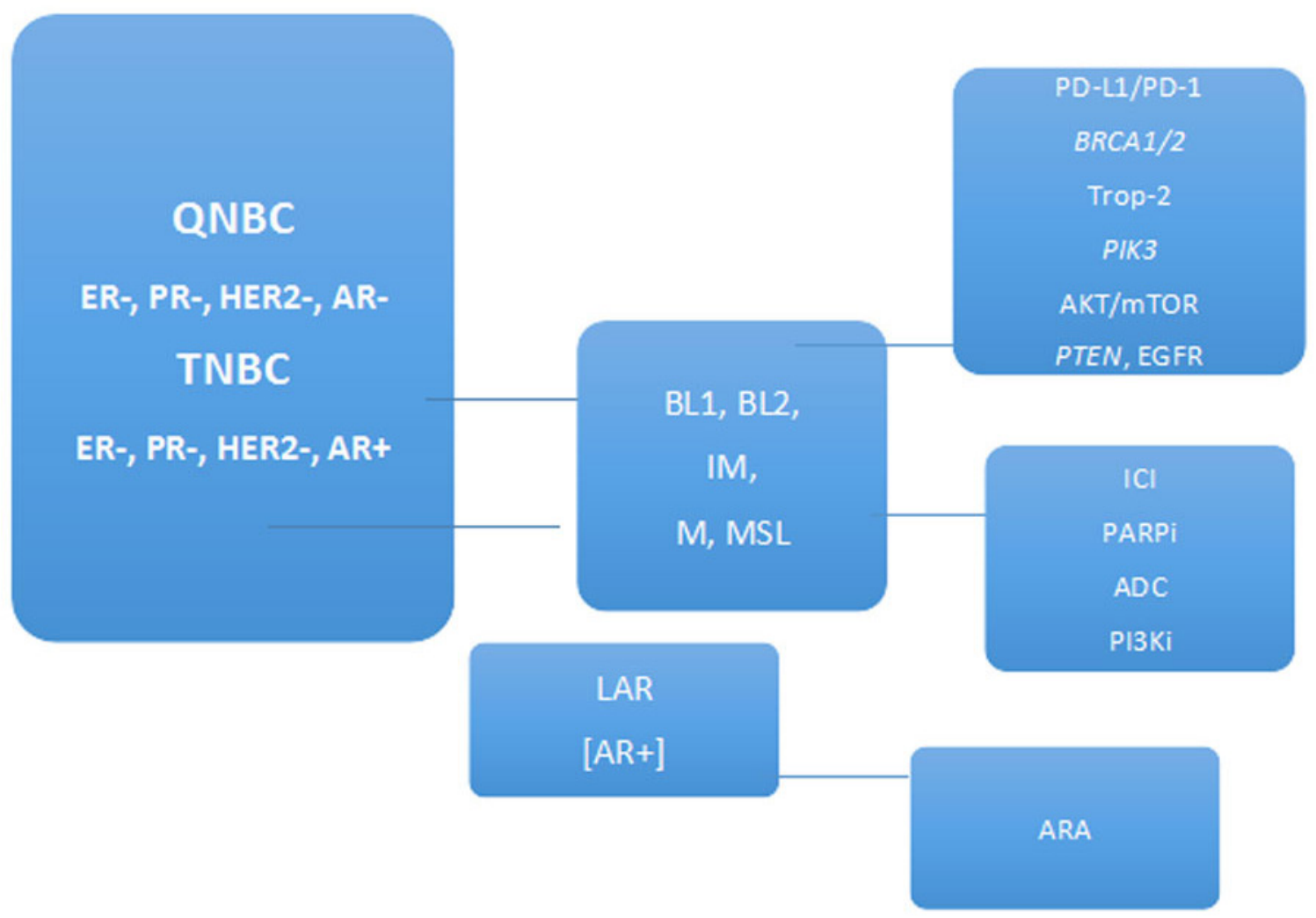

Figure 2. Molecular subtypes, therapeutic targets, and treatment strategies for patients with Triple-negative breast cancer (TNBCs) and Quadruple-negative breast cancer (QNBC) [3, 5, 6]. (Receptors: AR, Androgen receptor; ER, Estrogen receptor; HER2, Human epidermal growth factor receptor 2; PR; Progesterone receptor; Molecular subtypes: BL, Basal-like; IM, Immunomodulatory; LAR, Luminal androgen receptor; M, Mesenchymal; MSL, Mesenchymal stem-like; Therapeutic targets: PD-L1, programmed death ligand 1; PD-1, programmed cell death protein-1; BRCA1/2, tumor suppressor genes; Trop-2, Trophoblast cell-surface antigen; PI3K, phosphoinositide-3 kinase; AKT/mTOR, protein kinase B/the mammalian target of rapamycin; PTEN, phosphatase and tensin; EGFR, epidermal growth factor receptor; Treatment strategies: ICI, immune checkpoint inhibitors; PARPi, Poly ADP-ribose polymerase (PARP) inhibitors; ADC, Antibody drug conjugate; PI3Ki, PI3K inhibitors; ARA, AR antagonists).

revealed certain beneficial outcomes [26-31]. As a result, many these therapeutic options have been approved by the US Food and Drug Administration (FDA) as targeted therapies for selected subsets of patients with TNBC (Table 1) [26-31].

To fill an unmet medical need for a life-threatening disease like metastatic TNBC, on March 11, 2019, the U.S. FDA granted the accelerated approval for atezolizumab (Tecentriq), a programmed death ligand-1 (PD-L1) inhibitor, in combination with CHT agent, nab-paclitaxel (Abraxane), for the treatment of patients with PDL1-positive, unresectable or metastatic TNBC (based on the IMpassion130 trial) [26].

In addition, on November 13, 2020, the FDA granted the accelerated approval to pembrolizumab (Keytruda) in combination with CHT for the treatment of patients with locally recurrent unresectable or metastatic TNBC (based on the KEYNOTE-355 trial), whose tumors express PD-L1 (combined positive score (CPS) $\geq 10$ ) as determined by an FDA approved test [28].

Furthermore, on April 7, 2021, the FDA granted the regular approval to sacituzumab govitecan (Trodelvy) for patients with unresectable locally advanced or metastatic TNBC, who have received two or more prior systemic therapies (at least one of them for was for the metastatic disease) (based on the ASCENT trial) [31].

It should be highlighted that the combinations of certain therapeutic agents from various pharmacologic classes and sequences, in which they are used, can be crucial to "awakening" of the antitumor immune response of the host [32]. As a consequence, such a strong response may help in the transformation of immunologically "cold" into "hot" tumor areas in the metastatic TNBC [32]. Due to rapid advances in this field, many women with metastatic, previously incurable TNBC may now have some reasonable hope that the durable responses to ICI can be achieved [33]. Such a progress may happen, especially when the ICI are used in combination with CHT, depending on the individual patient scenario, in which the immune system can be invigorated [33].

Immune checkpoint inhibitors (ICI) (atezolizumab and pembrolizumab) 


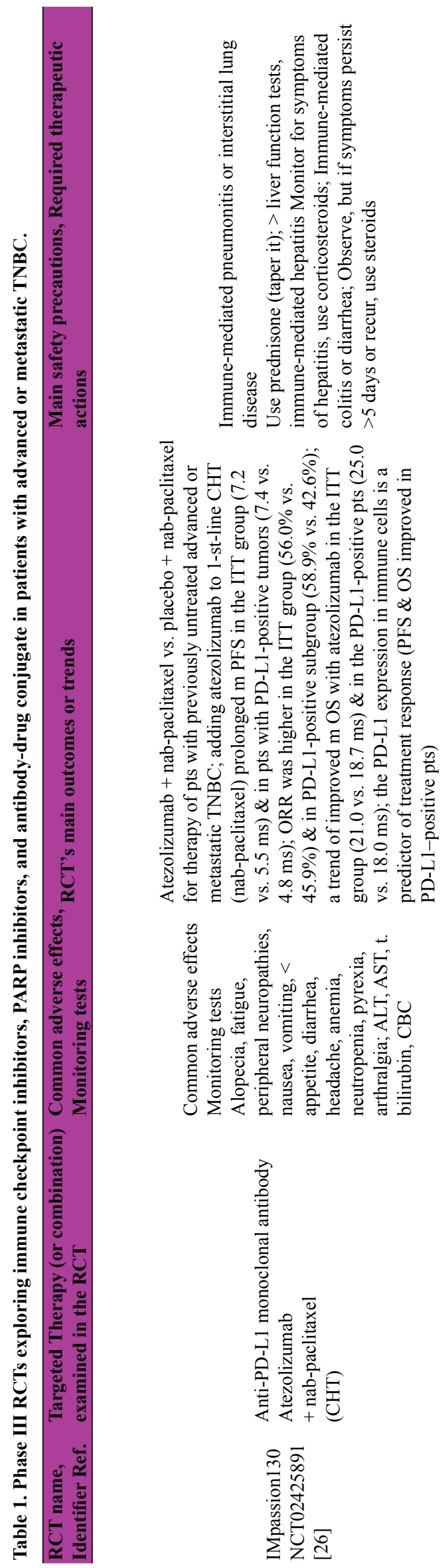

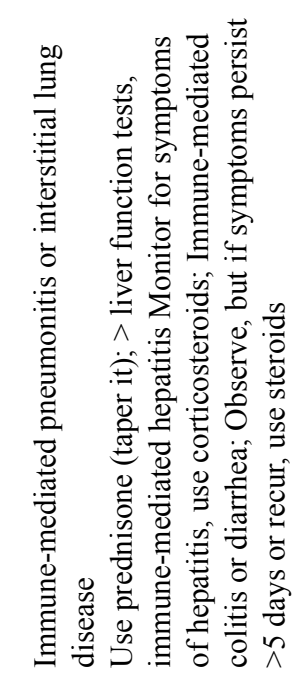

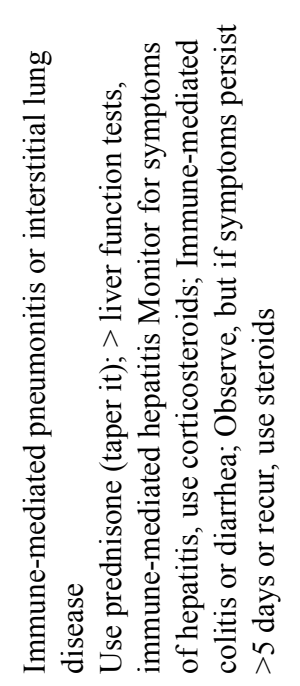

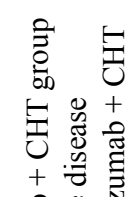

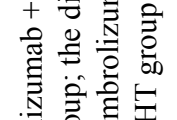

총 言总它

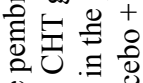

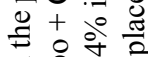

$\exists$ 造实

市

它导总

胥 $\Xi$

त्ञ

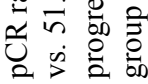

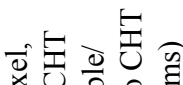

局

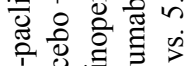

迹

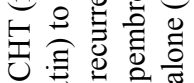

+ 등

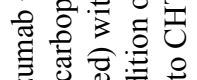

워

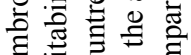

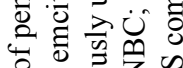

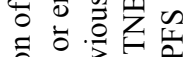

可产总

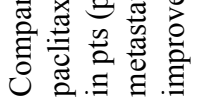

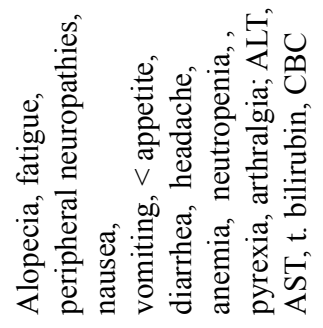

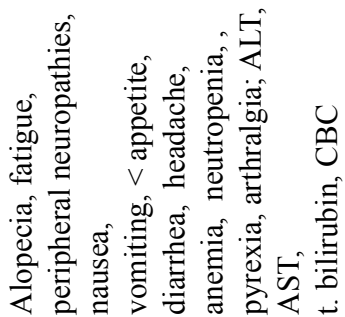

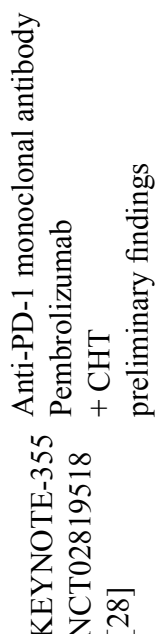




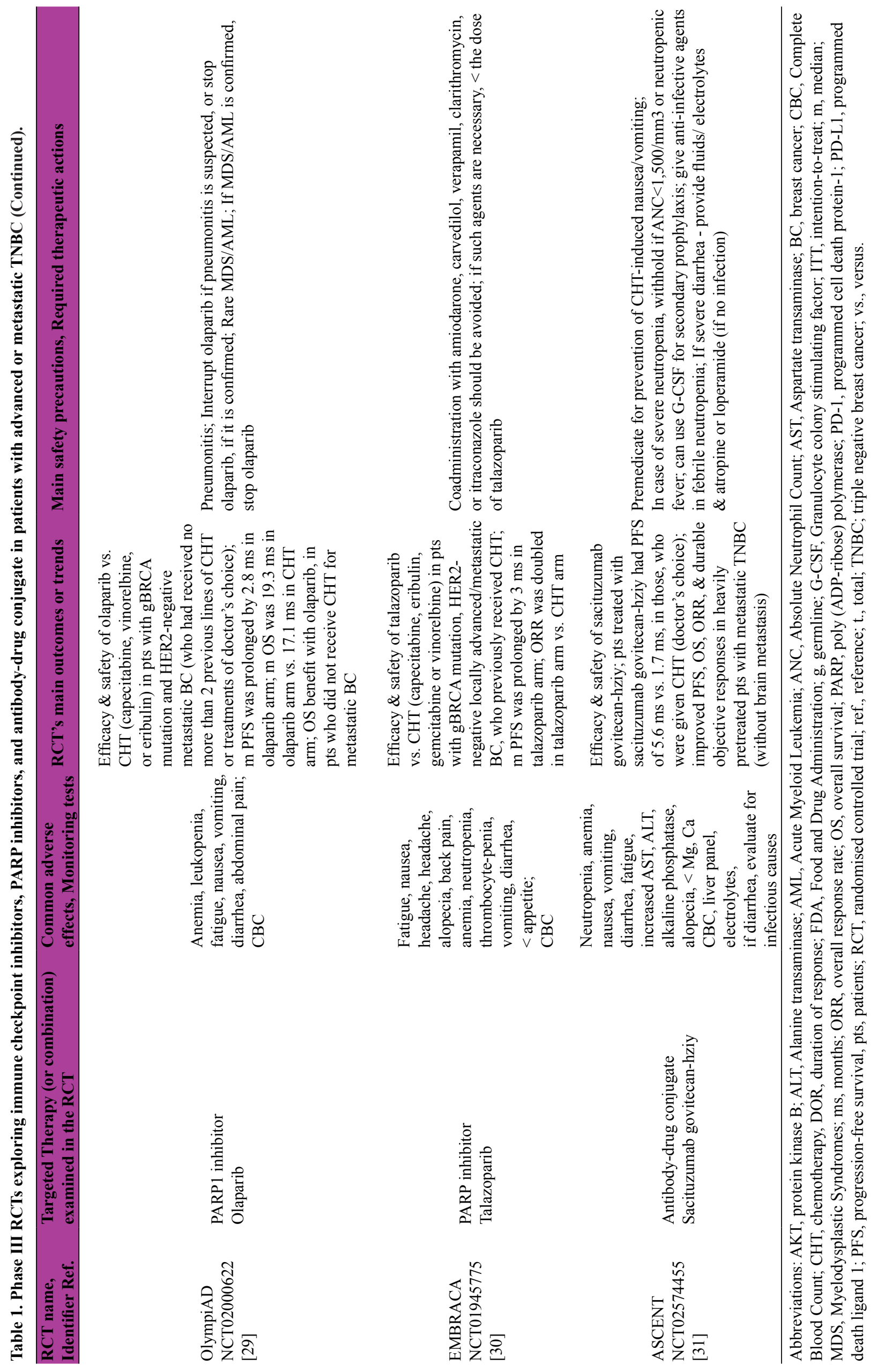


According to recent RCTs data, ICI that target the programmed death ligand-1 (PD-L1) and programmed death receptor-1 (PD1) have shown clinical benefits for patients with advanced and metastatic TNBC (e.g., in combination with CHT) (Table 1) [26, 27].

In TNBC, PD-L1 is usually expressed on the tumor-infiltrating immune cells (TILs), and malignant tumor cells, while the PD-1 is commonly expressed on T-cells [26, 27]. When the PD-L1 binds to PD-1, an inhibitory signal is being send that causes the T-cell suppression [34]. To counteract the undesirable immune system suppression, ICI, such as the PD-L1 inhibitor, atezolizumab, or the PD-1 inhibitor, pembrolizumab, in combination with CHT, can be considered to treat women with locally advanced, recurrent, and metastatic TNBC (rather earlier than later in the disease course) $[26,27]$. In fact, elevated TILs or expression of PD-L1/ PD-1 immune checkpoint complexes is often related to a higher anti-tumor immunity, and a better patient prognosis [33]. This can be considered a biomarker, detecting potential candidates for treatment with ICI [33]. PD-L1, which is expressed in about $40 \%$ of TNBC, has become a novel therapeutic target, especially in metastatic TNBC $[32,33]$.

The IMpassion130 trial illustrates the favorable effects of adding atezolizumab (an anti-PD-L1 monoclonal antibody), to nabpaclitaxel (CHT) (comparing to nab-paclitaxel alone, as a first-line therapy) for women with metastatic TNBC (Table 1) [26].

Similarly, the KEYNOTE-522 trial has explored the addition of pembrolizumab (an anti-PD-1 monoclonal antibody) to CHT (neoadjuvant $\mathrm{CHT}$, and continued adjuvant $\mathrm{CHT}$ ) in untreated patients with stage II or III TNBC, showing some promising effects (Table 1) [27]. It should be highlighted that in contrast to the IMpassion 130 trial, the addition of pembrolizumab to standard CHT in the KEYNOTE-522 trial has revealed improvements in pCR [27]. Likewise, the ongoing KEYNOTE-355 trial, investigating the addition of pembrolizumab to CHT, among patients with untreated, locally recurrent, inoperable or metastatic TNBC, has revealed some encouraging results (Table 1) [28].

\section{Poly-ADP-ribose polymerase (PARP) inhibitors (olaparib and talazoparib)}

Based on recent RCTs, some PARP inhibitors, targeting specific genetic mutations or molecular signaling pathways (which govern malignant cells growth), have been applied in monotherapy, or in combination with CHT, in patients with metastatic TNBC $[29,30]$. BRCA1 and BRCA2 are tumor suppressor genes that play a key role in the repair of DNA breaks [35]. The BRCA1/2 mutations are related to increased familial inheritance, early-onset, high tumor aggression, and poor TNBC prognosis [35]. As a result of these loss of function mutations, TNBC tumors that carry the mutated BRCA1/2 genes, are unable to repair their damaged DNA [35]. In these circumstances, blocking of the PARP enzymes, via the PARP inhibitors causes the lethal accumulation of irreparable DNA breaks, and cytotoxic PARP-DNA complexes in the tumor [36]. Recently PARP inhibitors (olaparib and talazoparib) have been approved as targeted therapy that can be used in almost $20 \%$ of patients with TNBC (who have germline BRCA1/2 mutations) $[29,30]$.

The OlympiAD trial has explored the efficacy of olaparib, in patients with metastatic, germline BRCA mutated, HER2-negative $\mathrm{BC}$, who had received no more than two previous lines of CHT [29]. According to this study results, olaparib monotherapy revealed beneficial effects, compared to standard CHT (e.g., median PFS was almost three months longer and the risk of disease progression or death was over $40 \%$ lower in women, who received olaparib, compared to those who received standard CHT (Table 1) [29].

Similarly, the EMBRACA trial examined the efficacy of talazoparib, in women with advanced BC and germline BRCA1/2 mutations (who previously received CHT) [30]. The EMBRACA trial has shown that patients who were treated with talazoparib had improved PFS compared to those, who received CHT (Table 1) [30]. Moreover, the most favorable responses were reported in a subset of patients with TNBC, who harbored germline BRCA1/2 mutations. In addition, PARP inhibitors, used in combination with CHT, can be particularly helpful in the treatment of breast tumors with not only germline BRCA1/2 mutations, but also with PALB2, RAD51, p53, and CHEK2 gene mutations [37].

\section{Anti-Trop-2 antibody-drug conjugate (ADC) (Sacituzumab govitecan-hziy)}

Trophoblast cell-surface antigen (Trop-2) is a glycoprotein overexpressed in several epithelial cancers, including TNBC, in which it plays a role of the growth-stimulating signal [31]. Sacituzumab govitecan-hziy is an antibody-drug conjugate (ADC) that combines a humanized monoclonal antibody that targets the Trop-2 with an active metabolite of irinotecan, which is a topoisomerase I inhibitor ( $\mathrm{SN}-38)$, via the cleavable linker [31]. Upon binding to Trop-2, the SN-38 is transported to the breast tumor cells. Subsequently, via the cleavable linker, the SN-38 is being deployed into the tumor itself and the tumor's microenvironment (TME) [31].

According to the ASCENT trial, the efficacy and safety data of sacituzumab govitecan-hziy (e.g., improved PFS, OS, and durable objective response (DOR)) in heavily pretreated patients with metastatic TNBC have been revealed (Table 1) [31]. Sacituzumab govitecan has been the first ADC, approved for patients with relapsed or refractory metastatic TNBC (who have failed two prior CHTs) [31].

\section{Future directions - Selected actionable pharmacologic targets and emerging therapies}

There are several emerging therapies, which target the tumordriving signaling networks in TNBC. For instance, it has been reported that the phosphoinositide-3 kinase (PI3K) and protein kinase $\mathrm{B}$ (PKB or AKT) signaling pathways may represent novel actionable targets in TNBC. In fact, in approximately one fourth of primary TNBCs, activating mutations (e.g., PIK3CA and AKT1) occur in these pathways [38]. In an attempt to solve this challenge, PI3K inhibitors have been examined in RCTs, and revealed some benefits in patients with advanced TNBC, in whom breast tumors harbored PIK3CA mutations [38]. In addition, the PI3KCA inhibitor, alpelisib, has improved PFS in patients with hormone receptor (HR)-positive, HER2-negative, PIK3CA-mutated advanced or metastatic BC [39].

Similarly, the AKT inhibitors (e.g., ipatasertib and capivasertib) have shown favorable clinical effects with regard to patients with high-risk TNBC [40, 41]. In particular, in the LOTUS trial (a phase II RCT) patients with advanced or metastatic TNBC were treated with ipatasertib and paclitaxel vs. placebo and paclitaxel. The LOTUS study has reported that women with metastatic TNBC, in the ipatasertib arm had an improved median PFS, compared to the placebo arm (6.2 months vs. 4.9 months) [40]. Notably, in the subset of patients with metastatic TNBC and PIK3CA/AK1/PTEN mutations, women treated with ipatasertib (and paclitaxel) had a median PFS of 5.3 months vs. 3.7 months in those, who received the placebo (and paclitaxel) [40].

Likewise, the PAKT trial (a phase II RCT) has examined the efficacy of another AKT inhibitor, capivasertib, in combination with the same CHT agent (paclitaxel), compared to a placebo and paclitaxel, in a group of women with untreated metastatic TNBC [41]. Based on the PAKT study report, an addition of capivasertib 
to paclitaxel resulted in improving the median PFS (5.9 months vs. 4.2 months) and OS (19.1 months vs. 12.6 months) compared to the paclitaxel alone arm. It should be highlighted that the advantages of capivasertib (with paclitaxel) were even more evident in the subset of women with TNBC, whose tumors harbored PIK3CA/ AK1/PTEN mutations (e.g.,. among these particular patients, a median PFS was 9.3 months vs. 3.7 months, in those, who were treated with paclitaxel only) [41].

Notably, the mesenchymal (M) subtype of TNBC has been related to abnormal PI3K/ mammalian target of rapamycin (mTOR) pathway activation and poor clinical outcomes. In this context, adding the mTOR inhibitors (e.g., temsirolimus or everolimus) has been examined, in combination with CHT (e.g., a liposomal formulation of doxorubicin) and an anti-vascular endothelial growth factor (VEGF) antibody (bevacizumab) [42]. However, these agents are still undergoing clinical investigations, and they have not been incorporated to the standard of care TNBC treatment.

Current research progress on the therapeutic targets of chemotherapy agents - A spotlight on tubulin polymerization inhibitors: eribulin and utidelone

In line with the 4th European School of Oncology (ESO) and European Society for Medical Oncology (ESMO) International Consensus Guidelines for Advanced Breast Cancer (ABC 4), a sequential single-agent $\mathrm{CHT}$ has been recommended as the first choice for women with advanced $\mathrm{BC}$, and a combination therapy has been used predominantly for fast progression, visceral metastases, or urgent control of symptoms [43]. If a patient received previous anthracycline and taxane CHT and did not require combination treatment, a monotherapy (e.g., capecitabine, vinorelbine, or eribulin), has been the preferred option [43] According to the 5th ESO-ESMO international consensus guidelines for $\mathrm{ABC}$, in patients with advanced or metastatic TNBC (regardless of BRCA status), who received prior treatment with anthracyclines (with or without taxanes) in the (neo)adjuvant setting, carboplatin (that revealed comparable efficacy and a lower toxicity than docetaxel) represents a valid therapeutic strategy. For non-BRCA-associated advanced or metastatic TNBC, there are no data supporting specific CHT recommendations, besides platinum agents [44].

Unfortunately, some patients have developed resistance to anthracycline and taxanes, and thus, new approaches are needed. One of the promising CHT options consists of the application of tubulin polymerization blockers, such as eribulin and utidelone, which have been investigated in clinical studies [45, 46, 47]. Eribulin, a tubulin polymerization inhibitor, has demonstrated some favorable efficacy and safety, in patients with advanced TNBC (e.g., significantly prolonged OS compared to capecitabine, in advanced TNBC) [45]. Moreover, the PFS was improved with the use of eribulin, compared to vinorelbine, in women with locally recurrent or metastatic BC [46]. Utidelone, a non-taxane tubulin polymerization inhibitor, is a genetically engineered analogue of epothilone. A recent trial comparing the efficacy and safety of utidelone plus capecitabine with the efficacy and safety of capecitabine alone, in patients with metastatic $\mathrm{BC}$ (who progressed after previous CHT with anthracycline and taxane) has shown the median PFS of 8.4 months in the combination therapy group and 4.3 months in the monotherapy group [47]. In addition, a prolonged PFS was reported with the combination therapy compared to the monotherapy, in the subgroup of women with advanced TNBC [47].

\section{Conclusion}

In summary, the majority of TNBCs are characterized by a negative or very low AR expression. The AR-negative TNBC represents a highly aggressive QNBC phenotype. Presumably, placing QNBC into a distinct category from the TNBC subtype could be a reasonable step forward. Unfortunately, the absence of expression of ER, PR, AR, and HER2 has reduced, for many years, the TNBC and QNBC pharmacologic treatment options to cytotoxic CHT. However, at present, some novel therapies, which target the host immune tumor surveillance system (e.g., via PDL1 and PD-1 ICI, such as atezolizumab and pembrolizumab), the damaged DNA repair machinery secondary to BRCA1/2 mutations (e.g., by PARP inhibitors, like olaparib and talazoparib), and the Trop-2 (e.g., via an ADC, sacituzumab govitecan-hziy) represent desirable breakthrough strategies.

The current status of research on synergistic treatments of these malignancy can be summarized, as follows: the immunotherapy for immune checkpoints, molecular targeted therapies, and new CHT strategies should be offered for many eligible patients with advanced and metastatic TNBC. Of course, such therapies need to be precisely tailored to each individual patient's clinical scenario, and then monitored closely.

With regard to the immunotherapy, atezolizumab plus nabpaclitaxel can be used as the first-line therapy for PD-L1-positive advanced or metastatic TNBC (e.g., de novo or at least one year since (neo)adjuvant CHT). However, ICI monotherapy in later lines for advanced or metastatic TNBC is not recommended, because of low response rates. Importantly, evaluating the PDL1 status, with the Ventana (SP142) test (a companion diagnostic immunohistochemistry (IHC) assay) for the use of atezolizumab (in combination with a taxanes) for the first-line therapy in advanced or metastatic TNBC (a cut-off of $1 \%$ positive staining on immune cells) is crucial to optimize the patient's selection for this treatment.

In women with advanced or metastatic TNBC, who carry BRCA1/2 mutation, the targeted treatment with PARP inhibitors (e.g., olaparib and talazoparib) can be beneficial. In order to take advantage of these therapies, such patients need to be tested for the presence of germline BRCA $1 / 2$ mutations (to determine whether or not they would qualify for this option).

The AR is a potential target in TNBC, and thus, the targeted application of AR antagonists (e.g., bicalutamide, enzalutamide, or abiraterone acetate) has been explored in clinical studies, which suggested that these agents have antitumor activity and good tolerance in patients with AR-positive TNBC subtype (however, at present AR antagonists should not be used in the clinical setting, since a standardized assay for the AR is still not available)

Moreover, in the CHT armamentarium against advanced or metastatic TNBC, eribulin appears superior to paclitaxel, carboplatin, capecitabine, and vinorelbine options. Likewise, a combination treatment of utidelone and capecitabine seems to be superior to capecitabine alone in the refractory forms of TNBC. Also, in recent clinical studies, AKT inhibitors, ipatasertib, and capivasertib have shown some good efficacy and safety results, in patients with advanced TNBC.

It should be highlighted that disseminating current knowledge about promising research avenues and clinical considerations in the TNBC and QNBC area within the medical communities (e.g., among the physicians and their patients at risk, or afflicted by this malignancy) is crucial, since it will maximize chances for the improved patient's outcomes.

\section{Author contributions}

The author Katarzyna Rygiel contributed solely to the work.

\section{Conflicts of interest}

The author declares that she has no conflicts of interest. 


\section{Ethical approval}

Not applicable.

\section{References}

1. Garrido-Castro AC, Lin NU, Polyak K. Insights into molecular classifications of triple-negative breast cancer: improving patient selection for treatment. Cancer Discov 2019; 9: 176-198.

2. Kohler BA, Sherman RL, Howlader N, Jemal A, Ryerson AB, Henry KA, et al. Annual report to the nation on the status of cancer, 19752011, featuring incidence of breast cancer subtypes by race/ethnicity, poverty, and state. J National Cancer Institute 2015; 107 (6): djv048.

3. Gupta GK, Collier AL, Lee D, et al. Perspectives on triple-negative breast cancer: current treatment strategies, unmet needs, and potential targets for future therapies. Cancers (Basel) 2020; 12: 1-33.

4. Siddharth S, Sharma D. Racial disparity and triple-negative breast cancer in African-American women: a multifaceted affair between obesity, biology, and socioeconomic determinants. Cancers (Basel) 2018; 10: 1-19.

5. Bhattarai S, Saini G, Gogineni K, Aneja R. Quadruple-negative breast cancer: novel implications for a new disease. Breast Cancer Res 2020; 22 (1): 127. doi: 10.1186/s13058-020-01369-5.

6. Lehmann BD, Jovanović B, Chen X, Estrada MV, Johnson KN Shyr Y, et al. Refinement of triple-negative breast cancer molecular subtypes: implications for neoadjuvant chemotherapy selection. PLoS One 2016; 11(6): e0157368.

7. Burstein MD, Tsimelzon A, Poage GM, Covington KR, Contreras A, Fuqua SA, et al. Comprehensive genomic analysis identifies novel subtypes and targets of triple-negative breast cancer. Clin Cancer Research 2015;21(7):1688-1698.

8. Echavarria I, López-Tarruella S, Picornell A, García-Saenz J, Jerez Y, Hoadley K, et al. Pathological response in a triple-negative breast cancer cohort treated with neoadjuvant carboplatin and docetaxel according to Lehmann's refined classification. Clin Cancer Res 2018; 24 (8): 1845-1852.

9. Sharma P. Biology and Management of Patients With TripleNegative Breast Cancer. Oncologist 2016; 21 (9): 1050-1062. doi:10.1634/theoncologist.2016-0067.

10. Bhattarai S, Klimov S, Mittal K, Krishnamurti U, Li XB, OpreaIlies G, et al. Prognostic role of androgen receptor in triple negative breast cancer: a multi-institutional study. Cancers 2019; 11 (7).

11. Elebro K, Bendahl PO, Jernström H, et al. Androgen receptor expression and breast cancer mortality in a population-based prospective cohort. Breast Cancer Res Treat 2017; 165: 645-657.

12. Peters AA, Buchanan G, Ricciardelli C, Bianco-Miotto T, Centenera MM, Harris JM, et al. Androgen receptor inhibits estrogen receptoralpha activity and is prognostic in breast cancer. Cancer Res 2009; 69 (15): 6131-6140.

13. Hon JD, Singh B, Sahin A, Du G, Wang J, Wang VY, et al. Breast cancer molecular subtypes: from TNBC to QNBC. Am J Cancer Res 2016; 6 (9): 1864-1872.

14. Gucalp A, Tolaney S, Isakoff SJ, Ingle JN, Liu MC, Carey LA, et al. Phase II trial of bicalutamide in patients with androgen receptorpositive, estrogen receptor-negative metastatic breast cancer. Clin Cancer Research 2013; 19 (19): 5505-5512.

15. TA, Miller K, Yardley DA, Eakle J, Schwartzberg LS, O'Shaughnessy J, et al. Enzalutamide for the treatment of androgen receptor-expressing triple-negative breast cancer. J Clin Oncol 2018, 36(9): 884-890.

16. Yu Q, Niu Y, Liu N, Zhang JZ, Liu TJ, Zhang RJ, et al. Expression of androgen receptor in breast cancer and its significance as a prognostic factor. Ann Oncol 2011; 22 (6): 1288-1294.

17. Bonnefoi H, Grellety T, Tredan O, Saghatchian M, Dalenc F, Mailliez A, et al. A phase II trial of abiraterone acetate plus prednisone in patients with triple-negative androgen receptor positive locally advanced or metastatic breast cancer (UCBG 12-1) Ann Oncol 2016; 27(5): 812-818.

18. Lim B, Seth S, Huo L, Layman RM, Valero V, Thompson AM, et al. Comprehensive profiling of androgen receptor-positive (AR+) triplenegative breast cancer (TNBC) patients (pts) treated with standard neoadjuvant therapy (NAT) +/- enzalutamide. J Clin Oncol 2020; 38(15_suppl): 517.

19. Dehm SM, Tindall DJ. Alternatively spliced androgen receptor variants. Endocr Relat Cancer 2011; 18 (5): R183-R196.

20. Antonarakis ES, Lu C, Wang H, Luber B, Nakazawa M, Roeser $\mathrm{JC}$, et al. AR-V7 and resistance to enzalutamide and abiraterone in prostate cancer. N Engl J Med 2014; 371 (11): 1028-1038.

21. Hickey TE, Robinson JL, Carroll JS, Tilley WD. Minireview: the androgen receptor in breast tissues: growth inhibitor, tumor suppressor, oncogene? Molecular Endocrinol 2012; 26(8): 1252-1267.

22. Barton VN, Christenson JL, Gordon MA, Greene LI, Rogers TJ, Butterfield K, et al. Androgen receptor supports an anchorageindependent, cancer stem cell-like population in triple-negative breast cancer. Cancer Res 2017; 77(13): 3455-3466.

23. Gass P, Lux MP, Rauh C, Hein A, Bani MR, Fiessler C et al. Prediction of pathological complete response and prognosis in patients with neoadjuvant treatment for triple-negative breast cancer. BMC Cancer 2018; 18, 1051.

24. Pelizzari G, Gerratana L, Basile D, Fanotto V, Bartoletti M, Liguori A et al. Post-neoadjuvant strategies in breast cancer: From risk assessment to treatment escalation. Cancer Treat Rev 2019; 72, 7-14.

25. Angajala A, Mothershed E, Davis MB, Tripathi S, He Q, Bedi D, et al. Quadruple Negative Breast Cancers (QNBC) Demonstrate Subtype Consistency among Primary and Recurrent or Metastatic Breast Cancer. Transl Oncol 2019; 12 (3): 493-501.

26. Schmid P, Rugo HS, Adams S, et al. Atezolizumab plus nabpaclitaxel as first-line treatment for unresectable, locally advanced or metastatic triple-negative breast cancer (IMpassion130): updated efficacy results from a randomised, double-blind, placebo-controlled, phase 3 trial. Lancet Oncol 2020; 21: 44-59.

27. Schmid P, Cortes J, Pusztai L, McArthur H, Kümmel S, Bergh J et al. Pembrolizumab for early triple-negative breast cancer. N Engl J Med 2020; 382, 810-821.

28. Cortes J, Cescon DW, Rugo HS, Nowecki Z, Im SA, Yusof MM, et al. KEYNOTE-355 Investigators. Pembrolizumab plus chemotherapy versus placebo plus chemotherapy for previously untreated locally recurrent inoperable or metastatic triple-negative breast cancer (KEYNOTE-355): a randomised, placebo-controlled, double-blind, phase 3 clinical trial. Lancet 2020; 396(10265): 1817-1828.

29. Robson ME, Tung N, Conte P, Im SA, Senkus E, Xu B, et al. OlympiAD final overall survival and tolerability results: Olaparib versus chemotherapy treatment of physician's choice in patients with a germline BRCA mutation and HER2-negative metastatic breast cancer. Ann Oncol 2019; 30(4): 558-566.

30. Litton JK, Rugo HS, Ettl J, et al. Talazoparib in patients with advanced breast cancer and a germline BRCA mutation. N Engl J Med 2018; 379: 753-763.

31. Bardia A, Mayer IA, Vahdat LT, et al. Sacituzumab Govitecan-hziy in Refractory Metastatic Triple-Negative Breast Cancer. N Engl J Med 2019; 380 (8): 741-751.

32. Killock, D. Chemotherapy as a TONIC to invigorate PD-1 inhibition in TNBC. Nat Rev Clin Oncol 2019; 16, 464.

33. Thomas R, Al-Khadairi G, Decock J. Immune Checkpoint Inhibitors in Triple Negative Breast Cancer Treatment: Promising Future Prospects. Front Oncol 2021; 10: 600573.

34. Marra A, Viale G, Curigliano G. Recent advances in triple negative breast cancer: The immunotherapy era. BMC Med 2019; $17,90$.

35. Hatano Y, Tamada M, Matsuo M, Hara A. Molecular trajectory of BRCA1 and BRCA2 mutations. Front Oncol 2020; 10, 361.

36. McCann KE, Hurvitz SA. Advances in the use of PARP inhibitor therapy for breast cancer. Drugs Context 2018; 7, 1-30. 
37. Shi Y, Jin J, Ji W, Guan X. Therapeutic landscape in mutational triple negative breast cancer. Mol Cancer 2018; 17, 99.

38. Pascual J, Turner N. Targeting the PI3-kinase pathway in triplenegative breast cancer. Ann Oncol 2019; 30, 1051-1060.

39. André F, Ciruelos E, Rubovszky G, Campone M, Loibl S, Rugo HS et al. Alpelisib for PIK3CA-mutated, hormone receptor-positive advanced breast cancer. N Engl J Med 2019; 380, 1929-1940.

40. Kim SB, Dent R, Im SA, et al. Ipatasertib plus paclitaxel versus placebo plus paclitaxel as first-line therapy for metastatic triplenegative breast cancer (LOTUS): a multicentre, randomised, doubleblind, placebo-controlled, phase 2 trial. Lancet Oncol 2017; 18: 13601372.

41. Schmid P, Abraham J, Chan S, et al. Capivasertib plus paclitaxel versus placebo plus paclitaxel as first-line therapy for metastatic triple-negative breast cancer: the PAKT trial. J Clin Oncol 2020; 38: 423-433.

42. Basho RK, Gilcrease M, Murthy RK, Helgason T, Karp DD, MericBernstam F et al. Targeting the PI3K/AKT/mTOR pathway for the treatment of mesenchymal triple-negative breast cancer. JAMA Oncol 2017; 3, 509-515.

43. Cardoso F, Senkus E, Costa A, et al. 4th ESO-ESMO International Consensus Guidelines for Advanced Breast Cancer (ABC 4). Ann Oncol 2018; 29(8): 1634-1657.

44. Cardoso F, Paluch-Shimon S, Senkus E, et al. 5th ESO-ESMO international consensus guidelines for advanced breast cancer (ABC 5). Ann Oncol 2020; (12): 1623-1649.

45. Pivot X, Marmé F, Koenigsberg R, Guo M, Berrak E, Wolfer A Pooled analyses of eribulin in metastatic breast cancer patients with at least one prior chemotherapy. Ann Oncol 2016; 27(8): 1525-1531.

46. Yuan $\mathrm{P}, \mathrm{Hu} X$, Sun $\mathrm{T}$, et al. Eribulin mesilate versus vinorelbine in women with locally recurrent or metastatic breast cancer: A randomised clinical trial. Eur J Cancer 2019; 112: 57-65.

47. Zhang $\mathrm{P}$, Sun $\mathrm{T}$, Zhang Q, et al. Utidelone plus capecitabine versus capecitabine alone for heavily pretreated metastatic breast cancer refractory to anthracyclines and taxanes: a multicentre, open-label, superiority, phase 3, randomised controlled trial. Lancet Oncol 2017; 18(3): 371-383. 